\title{
Probability learning in the goldfish: I. Aversive reinforcement'
}

FORREST W. YOUNG AND HARMAN V. S. PEEKE UNIVERSITY OF SOUTHERN CALIFORNIA
Two groups of goldfish were run 200 trials in a shuttlebox; consistently shocking one side for one group, and shocking one side $80 \%$ of the time and the opposite side $20 \%$ for the second group. The $100 \%$ group chose the nonshocked side with a $100 \%$ frequency at asymptote. The $80 \%$ group chose the least frequently shocked chamber with a frequency significantly lower than the $100 \%$ group, but significantly greater than $80 \%$. Difficulties for several theories of probability learning are discussed.

The purpose of this experiment was to study the probability learning behavior of the goldfish with aversive reinforcement. The familiar mathematical learning models (Estes, 1950; Bush \& Mosteller, 1955) predict that an organism will match the probability of occurrence of the positive event; i.e., in the case where an aversive stimulus such as shock occurs $20 \%$ of the time in the right-hand compartment of an aquatic shuttlebox, the fish should after a sufficiently large number of trials, choose that side on $80 \%$ of the trials. Behrend \& Bitterman (1962), using positive reinforcement and a special correction method, were able to support the matching prediction with the African Mouthbreeder (Tilapia macrocephala), although they found that the fish maximized without the correction procedure. Based on these results Bitterman (1965) concludes that fish tend to probability match.

Method

Twenty goldfish, $1-1 / 2$ to $2-1 / 2$ in. long were used as Ss.

The apparatus was a Lafayette Aquatic Conditioning Apparatus. It consisted of a glass aquarium (6-1/2 $x$ $3-1 / 2 \times 3$ in.) divided into two $3 \times 3 \times 3$ in. compartments by a piece of opaque plastic with a 2 in. square doorway cut in the center. The right compartment's back and side walls were covered with black material, and the left compartment's back and side walls were covered with white material. The covering reduced distracting stimulation and served to differentiate the two compartments. The uncovered front glass allowed the $\mathrm{E}$ to observe the S's behavior. The floor of each compartment consisted of a stainless plate that functioned as an electrode. The other electrode for each side was a screenwire plate suspended just under the surface of the water. A control panel provided the $E$ with the capability of choosing both the level of shock and the compartment shocked. A warning stimulus was provided by a 40-watt bulb in a spun aluminum hood suspended 8 in. above the center of the apparatus. The intertrial and interstimulus intervals were controlled by a Minarik rotary timer.
The Ss were divided into two groups of ten fish. Each $S$ was placed in the apparatus and allowed to adapt for $5 \mathrm{~min}$. After adaptation, 200 trials were administered with fixed intertrial interval of $6 \mathrm{sec}$. Each trial consisted of a 5-sec. "warning stimulus," followed immediately by a 1-sec. shock in one or the other compartment. The shock was $60 \mathrm{cps} A C$ which was adjusted for each $S$ during the first few trials to a level at which it elicited sudden motor movement, but was well below tetanizing levels. The range of shock levels was estimated between 5-15 volts. Care was taken to alternate the side of the apparatus in which the $\mathrm{S}$ was first placed, although there was no relationship between the side in which the $S$ was placed and the side $S$ was in during the first trial. All 200 trials were administered in one session. Five Ss were run with the black-right side punished on $100 \%$ of the trials, and five were run with the white-left side punished on $100 \%$ of the trials. This group is referred to as the "100-0 group."

The second group of $10 \mathrm{Ss}$, called the "'80-20 group," was run in the same manner as the 100-0 group, except that five Ss were run with the black-right side punished on $80 \%$ of the trials and the white-left side punished on $20 \%$. The percentages were reversed for the other five Ss. The series of left and right shocks was random with two constraints: (1) for every block of 10 trials there were two shocks on one side, and eight on the other; and (2) at least two trials had to intervene before the less frequent side was shocked again. The series was the same for all Ss.

The response scored was the side of the shuttlebox the S occupied at UCS onset.

The raw data from the 80-20 group were submitted to a computer program which computed the least squares solution to the prediction equation derived from Estes' (1950) model (E-model). This least squares solution was then subjected to a Monte Carlo program which in turn generated simulated data for $10 \mathrm{Ss}$ over 200 trials. This simulated group of Ss will be referred to as the $80-20 \mathrm{MC}$ group. For a general discussion of the Monte Carlo technique see Bush \& Mosteller (1955). Results

The E-model learning rate for the 80-20 group (assuming side preferences were balanced out by the experimental procedure) was determined to be .028 . The theory predicted $30 \%$ of the variance for this group. The raw data for each $S$ in all three groups were converted into proportions of each response taken over 20 blocks of 10 trials each. The last five blocks of proportions were then analyzed by the Kruskal-Wallis two-way analysis by ranks. In addition, the 100-0 and 


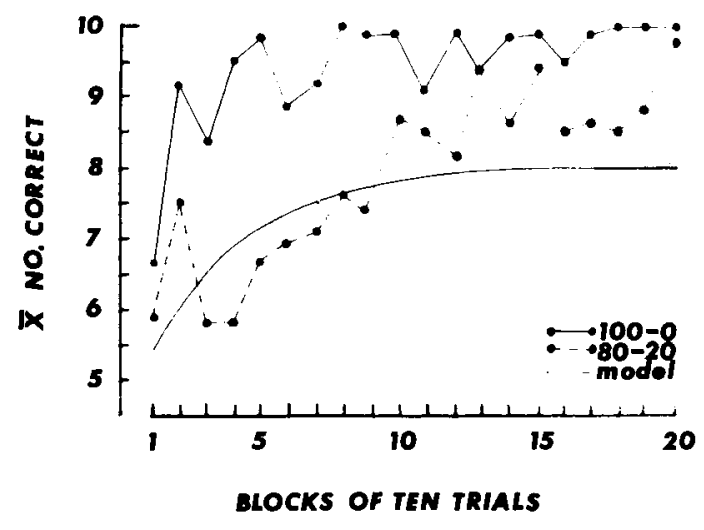

Fig. 1. Mean number of responses in the less frequently shocked compartment (correct responses) over trial blocks.

80-20 groups, and the 80-20 and 80-20MC groups were compared using the Mann-Whitney U-test. These non-parametric tests were chosen in favor of parametric tests because of the larger number of proportions equal to unity for the 100-0 group. Figure 1 presents these means and the stochastic learning model's least squares prediction.

The Kruskal-Wallis test indicated the three groups differ significantly for the last 50 trials $(p<.001)$. The two Mann-Whitney tests indicate the 80-20 group was significantly different from the 100-0 group ( $p<.01)$, and from the $80-20 \mathrm{MC}$ group $(p<.01)$. Again, these tests pertain to the last 50 trials.

Discussion

The statistical analysis indicates that the group of goldfish run under the 80-20 regimen neither matched nor maximized. This result is inconsistent with the E-model since it predicts probability matching (Estes, 1957). This prediction is independent of (1) all characteristics of the Ss; and (2) all aspects of the experimental situation excepting the frequency of shock.

Bush and Mosteller's model (BM-model), on the other hand, is capable of predicting our results, even though the most common assumptions (used to simplify the mathematics) developed predictions exactly the same as those of the E-model.2 An important and routinely used simplifying assumption implies that shock and no-shock have "equal but opposite" effects on the learning process (Bush \& Mosteller, 1955, p. 119). As these authors point out (1955, p. 288) this assumption does not appear intuitively reasonable and they show that it must be rejected when matching is not found.
For the 80-20 group, therefore, the effect of shock was not "equal but opposite" to the effect of no-shock. Unfortunately it is difficult to obtain a mathematically satisfactory estimate of the effects of shock and noshock (Bush \& Mosteller, 1955, p. 288) but it is possible to estimate the ratio of these two effects. This was done by using a BM-model formula (1955, p. 289) and assuming that the last 50 trials were asymptotic for this experiment. The procedure indicated that the behavioral effect of shock was 1.11 times greater than that for no-shock.

Using this ratio the BM-model can predict our results more accurately than the E-model; but note that the asset of more accurate prediction is balanced by the liability of having to estimate an extra parameter. For the E-model two parameters must be estimated from the data: The initial side preference of the Ss (if any); and the overall learning rate. For the BM-model three parameters must be estimated: The side preferences; the learning rate following shock; and the learning rate following no-shock. The extra parameter is necessary to accurately predict our results.

In addition, our results appear to restrict the generality of Bitterman's conclusion that fish probability match. With the aversive stimulus of shock, and without correction, our fish neither matched nor maximized; therefore, our results are not in conflict with the phyletic hierarchy postulated by Bitterman (1965). However, we must agree with Bitterman that his rat-fish dichotomy is strained by the data, and we feel that the addition of our data increases the strain.

\section{References}

Behrend, E. R., \& Bitterman, M. E. Probability-matching in the fish. Amet. J. Psychol., 1961, 74, 542-551.

Bitterman, M. E. Phyletic differences in learning. Amer. Psychologist, 1965, 20, 396-410.

Bush, R. R., \& Mosteller, F. Stochastic models for learning. New York: Wiley, 1955.

Estes, W. K. Toward a statistical theory of learning. Psychol. Rev., 1950, 57, 94-107.

Estes, W. K. Theory of learning with constant, variable, or contingent probabilities of reinforcement. Psychometrika, 1957, 22, 113-131.

\section{Notes}

1. The assistance of the University of Southern California Computer Sciences Laboratory and the Honeywell Corporation in providing computing time is gratefully acknowledged. The computer programs used in this study are available from the first author upon request.

2. Technically the E-model is the same as the E-controlled, equalalpha, zero-unity absorbing state BM-model. The BM-model referred to is the E-S-controlled, equal-alpha, zero-unity absorbing state BM-model. The rejected assumption is the equal-alpha assumption. Both the E-model and the BM-model are sets in the field of BMmodels. 